und 3 zu No. 29 des Trarifes in den Anmerkungen 1 und 2 za No. 237 des Entwurfes eines neuen Zolltarifes erhaiten sollen, noch Folgendes beifügen:

Die neue Fassung lebnt sich zwar dem Sinne nach vollständig der Anschanung an, die schon bisher in der Anmerkung 3 zu No. 29 Ausdruck gefunden hat. Hier. nach soll die in Aussicht gestellte Zollfreiheit keineswegs eine von dem Eintritte gewisser Bedingungen abhängige sein, sondern es soll vielmehr die Zollfreiheit bei Nichterfüllung gewisser Voraussetzungen wieder aufgehoben werden, d. h. die Bedingungen, unter denen Zollfreiheit zugesichert ist, sind keine aufschiebenden - sondern aufhebende - Bedingungen.

Hieran soll auch durch die neue Anmerkung 2 zu No. 237 des Entwurfes grundsätzlich nichts geändert werden, nur der Wortlaut ist ein anderer. Aber anch aus dem Wortlante der Anmerkung 2 zu No. 237 des Entwurfes geht meiner Anschaung nach keineswegs hervor, dass auf diesen in inländischen Betriebsanstalten gewonnenen zollbegünstigten Mineralolerzeugnissen an sich ein Zollanspruch haftet, der in Wegfall kommt, wenn die ordnungsgemässe Verwendung nicht nachgewiesen ist.

Es ist meiner Anschauung nach das Rechtsverhältnis vielmehr so aufzufassen, dass die bestimmt bezeichneten in inländischen Betriebsanstalten unter Ueberwachung gewonnenen Erzengnisse zollfrei sind, und dass vielmehr diese $Z$ ollfreiheit in Wegfall kommt, wenn es an irgend einem Erfordernis zur Beibehaltung dieser Zollfreiheit gebricht, sei es, dass das Erzengnis nicht richtig, d. h. zu einem nicht zollbegünstigten Zwecke verwendet wurde oder aber, dass zwar das richtige Erzeugnis richtig verwandt, die Verwendung aber nicht ordnungsmässig nachgewiesen ist. Bei dem Fortfall der demnach aufhebend bedingten Zollfreiheit tritt ron selbst die ursprüngliche vor der Bearbeitang bestandene Zollpflicht wieder in die Erscheinung.

Diese Anschauung wollte die Anmerkung 2 zu No. 237 des Entwurfes offenbar dadurch zum Ausdrucke bringen, dass sie im zweiten Satze die gewonnenen Erzeugnisse als a usländische behandelt wissen will.

Es soll dahin gestellt bleiben, ob es niclit vielleicht richtiger wäre, zu sagen:
Wird ron inländischen Betriebsanstalten zum Zwecke der Bearbeitung ausländisches Mineralöl bezogen, so kann eine Zollerleichterung insofern zugestanden werden, dass unter Beachtnng näher anzoordnender Ueberwachungs. massnahmen bei der Bearbeitung, von den hierbei gewonnenen Erzeugnissen die leichten Mineralöle, soweit sie nicht zu Schmier- oder Belenchtungszwecken ein. schliesslich der Erzeugung ron Leuchtgas verwendet werden, unter Ueberwachung der Verwendung suf Er. laubnisschein zollfrei belassen werden können.

So anfgefasst, würde sich vielleicht anch die Frage der Zollbehandlung der durch Unglücksfälle während der Versendung entstandenen $F$ ehlmengen leichter der oben vertretenen Lðsung näher bringen lassen.

Sind nämlich die in inländischen Betriebsanstalten gewonnenen Mineralolerzeugnisse zwar zollfrei, aber so, dass sie beim Eintritt ron gewissen aufhebenden $\mathrm{Be}$ dingungen wieder wie ursprünglich zollpflichtig werden, so ergiebt sich von selbst, dass, wean durch den einen oder anderen der beiden bei der mittelbaren Zollfreiheit Beteiligten - Betriebsanstalt, Gewerbsanstalt - der Eintritt der vorgesehenen aufhebenden Bedingungen herbeigeführt wird, die nunmehr wieder auflebende Zoll. pllicht nur dem aufzubürden wäre, dem der Eintritt jener Bedingung in irgend einer Weise zur Last gelegt werden kann.

Sofern dies aber weder bei dem einen noch dem anderen der Fall ist, dieser Eintritt vielmebr nur auf vom Willen dieser hier einzig und allein in Betracht kommenden beiden Arten von Beteiligten völlig unab hängigen Zufälligkeiten zurückzuführen ist, bleibt wohl kein anderer Weg übrig, weun snders nicht aus blos formellen Gründen eine übermässige Härte Platz greifen soll, als durch einen Zollerlass aus Billigkeit solchen be sonderen Verbältnissen gebührend Rechnung zu tragen.

Aus vorstehenden Erörterungen dürfte sich ergeben, dass der Bundesratsbeschluss rom 26. November 1896 im wesentlichen seine Aufgabe erfüllt; es erscheint aber wünschenswert, einige Mängel und Lücken, die sich bei dem Vollzuge dieser Bestimmungen herausgestellt haben, zu beseitigen bezw. zu ergänzen. Hierdnrch könnte manche zeitraubende Streitfrage beseitigt werden und die Gleichheit des Vollzuges und die Sicherheit des Gefălles nur gewinnen.

\title{
Beitrag zum Nachweise von mineralischen und organischen Verunreinigungen in Hartharzen.")
}

\author{
Von Dr. J. Hertkorn.
}

Die Untersuchung der Hartharze: halbweiche and harte Kopale, auf organische und mineralische Verunreinigungen, ist bisher im Fabrikbetriebe stets oberflächlich und anf mechanischem Wege ansgeführt worden. Diese Prüfung bestand lediglich in der Trennung durch Auslese der grösseren Stücke von den kleineren und Sieben der Splitter and les sog. Dust (Staubes) durch eine Anzabl Siebe ron bestimmter und verschiedener Maschenweite. Je nach der Menge der grösseren und kleineren Splitter und der Menge des Dust wurde der Handelswert der Kopalprobe taxiert. Die Kopalsplitter und -stücke sind als reiner Kopal angesehen worden, wahrend der Dust selten oder nicht einer Veraschung

*) Chemiker Zeltung 1902, No. 63. unterworfen warde. $\mathrm{Da}$ es an einer einfachen und zaverlussigen Methode fehlte, die organischen Verun. reinigungen aus den schwer löslichen Hartharzen glatt zo trennen, so nnterblieb die Bestimmuug derselben in den meisten Fällen. Bei den hohen Preisen dieser Hartharze, besonders derjenigen für Linoleum und Lack. zwecke, musste der Fabrikant sich mit den geringwertigen Dust- und Splitterkopalen und dergl. abfinden und diese an Stelle der Stückenkopale verwenden. Die ersteren sind indessen oft sebr unrein und erschweren dadurch anch noch die Weiterverarbeitung wesentlich. Als organische Verunreinigungen kommen hauptsächlich in Betracht: Rinden-, Wurzel-, Holz- und Blätterfragmente, sowie Humus und hamusăhnliche Stoffe; als mineralische Verunreinigungen: Sand, Lehm, Tou, Kalksteinfrag- 
mente, Kiesel, Mergel etc. Im Speziellen sind die Humus. substanzen, Blätter-, Rinden- and Holzteile für die Weiterverarbeitung der Hartharze durch Schmelzen and Ueberführnng in den löslichen Zustand sebr störend, da einerseits in der über $300^{\circ} \mathrm{C}$. heissen Schmelze diese Verunreinigangen verkohlen und das loslich gemachte Hartharz intensiv färben, ausserdem führt die Klärung eines solchen Harzes in Auflösung za grossen and oft nnüberwindlichen Schwierigkeiten. Aus diesem Grunde werden die gesiebten Kopaldust-Sorten einer trockenen oder nassen Separation (Schlämme) anterworfen, wobei die organischen nnd miveralischen Verunreinigungen von dem eigentlichen Hartharzstaube mehr oder weniger getrennt werden. Der Harzstaub wird event. noch bei höherer Temperatnr geblockt, $d$. h. gefrittet, and dadurch leichter und ohne Verlust versandfähig gemacht.

Die Veranreinigangen der Splitter- und Dust-Hartkopale schwanken ron 0,5 bis ca. $50 \%$; über $5 \%$ organische Verunreinigungen finden sich selten vor, das Mittel ist $0,3-2,5 \%$ und mineralische Stoffe ca. $1-5 \%$. Grobssere Verunreinigungen bestehen aus Sand and Erde. Da die Hartkopalsplitter und -dust am Produktionsorte oder in England oft der mechanischen Reinigung unterworfen werden, um an Fracht zu sparen, so trifft man heutzutage anf dem Kopalmarkte selten noch Splitterkopale an, welche grössere Verunreinigangen aufweisen. Leider lässt die Verpacknngsart noch sehr viel za wünscben übrig, indem durch die Sackpackung stets grossere Verluste an Dust oder Splitter anf dem Transporte vorkommen und die Ware durch die rohe Gewebefaser der Sacke verunreinigt wird.

Als Lösungsmittel der halbweichen und harten Kopale behufs der quantitativen Bestimmung der mineralischen und organischen Verunreinigungen kommen in Betracht: Aethylalkohol über 96 Gew.-Prozente, Propyl-, Butyl- und Amylalkohol, sowie die Acetate derselben besonders Amylacetat, event. noch Terpentinol, leichtes Kamphoröl und Aethylätber. Die Löslichkeit der Kopale in den genannten Losungsmitteln nimmt, wie bekannt, je nach der Art des Kopales, mit dem steigenden Moleknlargewichte der Alkohole and Ester zu, so dass der Amylalkohol für sich partiell leichter lösend wirkt als die niederen Glieder; am leichtesten auflösend wirken indessen die Acetate der höheren Alkohole, wie Propyl-, Butyl- und Amylacetat, und unter diesen wieder das leicht zugängliche und billige, wasserfreie Amylacetat des Handels in Mischnog mit Amplalkohol und hoch. prozentigem Aethylalkohol. Im allgemeinen wirkt jedes Lösungsmittel für sich weniger intensiv als die Mischung von Acetat mit Alkohol. Als zweckmässige and billige Mischung für den Zweck, verwendete ich 20-25 Teile Amylacetat, $40-50$ Teile Amylalkohol und 25-40 Teile über 96 gew.-prozent. Aethylalkohol. Die Extraktion im Soxhlet-Apparat führt nicht zum Ziel, indem dass feinst gepulverte Kopalharz schon bei gewöhnlicher Temperatur zusammenbackt and zähflüssige Schmiere bildet. Der Zusatz von einer gewogenen Menge ausgeglühten Seesandes zum Kopalharzpulver giebt ebenfalls nicht bessere Resultate. Es ist nämlich erkannt worden, dass selbst die heissen Kopallösungen schwer oder nicht filtrierbar sind und die Harze mit wenig Lösungsmitteln in der Wärme vollständiger in Lösung gehen, als solche mit einem grossen Ueberschusse davon, denn konzentrierte Hartkopalbarzlösungen geben anf weiteren Lðsungsmittelznsatz oft weisse Trübung oder Fallung and kolloidale Flüssigkeit, welche nicht mehr filtrierbar ist. Darch Aetberzusatz wird die Lösung meistens dünnflüssiger. Die in Anflösung befindlichen Hartharze wirken demnach in konzentrierten Lösungen wieder auflösend auf die sebwer oder nicht löslichen Bestandteile der Kopalharz. stoffe, so dass auf diese Weise die im reinen Lösnngs. mittel nicht löslichen Harzbestandteile in Lösung gebracht und gehsiten werden können. Versuche mit 10-20 prozent. Kolophoniumlösungen (amerikanisches Harz) in obigem Lösungsmittel geben dasselbe Resultat. Das amerikanische Harz wird bekanntlich bei der Herstellang von direkten Hartkopalbarzlösungen als Lösungsmittel in der Linoleum. und Lackbranche vielfältig verwendet. Solche Lösungen sind stets dünnflüssiger und weniger kolloidal and lassen sich leichter verarbeiten. Der Erfelg der vollständigen Auflösnng eines Hartkopalbarzes hängt somit von der richtigen Durchführung des ersten Lösungsmittelansatzes mit dem feinst gepulverten Hartkopalharze ab, indem man mit wenig Lösungsmittel im ersten Ansatze das Harz vollständig in Auflósung bringt.

Um das zeitranbende and oft nicht durchführbare Filtrieren von Hartkopalharzlösungen za umgehen, werden diese zweckmässig in der Warme vollständig absitzen gelassen und die klare Lösung vorsichtig von dem sandigen Bodensatze abgegossen. Wägbare Verluste an anlöslichem Rückstande sind nach meinen Versuchen nicht zu befürchten, indem höchstens einige Blattfragmente, welche nicht wägbar sind, mit der Dekantierflüssigkeit verloren gehen. Für die Bewertung eines Hartkopaldust oder Splitterkopales kommt es weniger auf 1 oder 2 Zehutel Prozente Unterschied in der Veranreinigung an, als auf die Art und Beschaffenheit derselben; von besonderer Wichtigkeit ist auch noch die Art und Beschaffenheit des zu untersuchenden Harzes selbst. Darüber giebt bekanntlich das Verhalten des Hartharzes gegenüber dem Lösungsmittel wertvollen Aufschluss, denn je härter und unverwitterter ein Kopaldust ist, desto langsamer und schwieriger gestaltet sich dessen Ueberfïhrung in den lóslichen Zustand. Die Auflösung ist unter denselben Umstïnden weit dickflussiger und in gewissen Grenzen gallertartiger, als bei den verwitterten und oxydierten minderwertigen Sorten. Die nachstehend beschriebene Methode ist einfach, billig und schnell zum Ziele führend und kann auch in jedem Fabrikslaboratorium leicht zar Ausführung gelangen. In ein nit Glasstab tariertes, $50-125 \mathrm{ccm}$ fassendes bohes Becherglas leichtester Sorte werden 5-10 g feinst gepulvertes, eventuell noch gebeuteltes Hartkopalharz genau abgewogen, mit 25-50 ccm des beschriebenen Lösungsmittels übergossen, wobei mit dem Glasstabe das Harzpulver mit dem Lösungsmittel ohne Unterbrechung gemischt und ersteres in Schwebe gebalten wird, bis eine Klumpenbildung nicht mehr zu befürchten ist. Sodann stellt man das Glas in Wasser von $70-80^{\circ} \mathrm{C}$., setzt das Umrübren bis zum angehenden Kochen des Lösanģmittels fort und digeriert bei bedecktem Glase unter zeitweiligem Umrühren ca. 1/2-1 Std. Ist sämtliches Kopalharz rerschwunden bezw. in Auflösung gegangen, erkenntlich an dem pulverförmigen, sandigen and nicht backenden Bodensatze und harzfreien Glasstab, so lässt man in der Wärme vollständig absitzen und dekantiert die klare Harzlösung vorsichtig in ein grösseres reines Becherglas, so lange die Lósung klar ablänft. Ist durch eine Unvorsichtigkeit oder durch Zufall die dekantierte Flüssigkeit mit dem Bodensatze veranreinigt, so lässt man diese Flüssigkeit ebenfalls absitzen und giebt den Bodensatz zax Hauptmenge des ersten zurück. Als Spülflïssigkeit dient das vorgeschriebene Lősangsmittel. $Z u$ dem Harzrückstande giebt man von nenem $25-50 \mathrm{ccm}$ Lösungsmittel und verfahrt wie zu Anfang - das Umrühren hat nar zeitweise zu geschehen - and wiederholt diese Manipulation so oft, bis ein Tropfen Dekantierflüssigkeit auf dem Platinblech beim Erhitzen des letzteren die Abwesenheit ron Harz anzeigt. Je nach der Art der Verunreinigung des Kopalharzstaubes verwendet man nach dem ersten direkten Abgiessen bei 
$105^{\circ} \mathrm{C}$. getrocknete and gewogene Filter fiir die Filtration der daraaf folgenden Spülflüssigkeit des Harzrückstandes. $\mathrm{Da}$ diese späteren und weniger Harz enthaltenden Lösungen sich schlechter klären und nicht bodensatzfrei abgiessen lassen, verwendet man gewogene Filter. Auf diesen Filtern darf indessen das Harz während der Operation nicht eintrocknen, indem anderenfalls die Poren des Papieres verstopft und nur durch langwieriges Spülen wieder unvollkommen zu öfinen sind. Den harzfreien Rückstand, sowie das Filter spült man noch zwei mal mit Aethyläther gut aus, giebt das Filter in das Becherglas zuriick, lässt den Aether an freier Luft verdunsten, trocknet sodann zuerst im offenen Trockenschranke und nachher im geschlossenen bei $105^{\circ} \mathrm{C}$. bis zur Gewichtskonstanz. In einigen Hartkopalsorten finden sich zuweilen porzellanartig aussehende, beim Erbitzen intensiv und charakteristisch riechende Harzfragmente, welche ausserordentlich hart sind and nur sehr schwer in Lösung gehen. Jedenfalls ist das Nachspülen mit Aether anch dann vorteilhaft, wenn darch denselben das hochflüchtige Lősungsmittel verdrängt and der Rückstand schneller zur Wägung gebracht werden soll. Ist der trockene, im Glase befindliche Harzrückstand nicht lose und pulverförmig, sondern klebt an den Glaswandungen teilweise noch fest, so muss die Extraktion mit dem Amplacetat oder mit Aether vervollständigt werden. Zur Aschenbestimmung benutzt man den vorliegenden Trockenrïckstand oder verascht das Kopalharz direkt im Platintiegel mit salpetersaurem Ammonium.

\section{BERICHTE.}

Das fette 0el der Samen von Lindera Benzoin. Von Charles E. Caspari. Lindera Benzoin, auch bekannt als Benzoin odoriferum oder Laurus Benzoin und gewohnlich Gewürzholz, Gewürzbusch oder Fieberbnsch genannt, wächst überall in den Vereinigten Staaten. Das Oel der Rinde und der $Z$ weige riecht nach Wintergrün, siedet zwischen 170 und $300^{\circ}$, besitzt ein spez. Gewicht von 0,923 und enthält $9-10 \%$ Methylsalicylat. Das Oel der Beeren enthält etwa $4 \%$ eines Oeles von kamphorartigem Geruch; spez. Gewicht 0,850-0,855, mit einem Siedepunkt von $160-270^{\circ}$. Die Blätter enthalten ungefähr $0,3 \%$ eines nach Lavendel riechenden Oeles vom spez. Gewicht 0,888. Vorliegende Untersuchung beschäftigt sich wit dem fetten Oel der Beeren und mit der Laurinsäure, welche in dem Oel als Glyzerid gefunden wurde. Von dem mit Aether aus den Kernen der Beeren extrahiertem Oel wurde ein ätherisches Oel mit Wasserdampf abdestilliert, aber in so geringer Menge, dass eine genauere Untersuchung desselben unmöglich war. Bei einer Oelgewinnung im Grossen darch Pressen and Extrahieren der Rückstände wurden im Ganzen $45,6 \%$ Oel erhalten. Das gewonnene Produkt stellte ein festes Fett dar von gelber Farbe and ziemlich kristallinischer Bescbaffenheit. Dasselbe schmilat bei $26^{\circ}$ und löst sich leicht in Aethylalkohol, Benzin und Aceton, schwieriger in wasserfreiem Methylalkohol. Seine alkoholische Lösung reagiert neutral. Die Verseifnngszahl ist 284,4 , die Reichert-Meisslsche Zahl 1,3. Nach den ausführlichen Versuchen des Verfassers besteht das fette Oel der Samen von Lindera Benzoin aus Glyzeriden der Caprin-, Laurinund Oelsäure und zwar herrscht die Laurinsäure vor.

(American. Chem. Journal n. Oil Paint u. Drug. Rep.)

Untersnchnng des Oeles der roten Hollanderbeere, Sambucas Racemosa arborescens" von H. G. Byers und $\mathrm{Pa}$ al Hopkins. An den westlichen Abhängen des Cascade-Gebirges und in den Niederungen um den Pnget Sund wachsen grosse Mengen von roten Hollunderbeeren, "welche wegen ihrer. Grösse als zu der Spezies Racemosa gehörig angesehen werden. Die Beeren sind karmoisinrot und dienen den Vögeln zar Nahrung. Sie enthalten ein gelbes Oel, welches Verf. einer Untersuchung unterzogen. Das mit Aether extrahierte Oel ist gelb, wird aber beim Stehen oder bei längerem Erhitzen auf dem Wasserbade dunkel. Das Oel besitzt ein spez. Gewicht von 0,9072 bei $15^{\circ}$. Es wird fest bei $-8^{\circ}$ und schmilzt bei etwa $0^{\circ}$. Einen bestimmten Siedepankt besitzt es nicht, da es sich bei der Destillation selbst bei einem Druck von nur $20 \mathrm{~mm}$ zersetzt. Es schien ein
Gemisch von Glyzeriden der Fettsäuren zu sein und enthält $6,65 \%$ freie Säure, berechnet als Oelsäure. Da es dem Olivenöl sehr gleicht, warden seine Konstanten sowie seine Zusammensetzung genau geprüft. Das Oel enthält $0,66 \%$ Unverseifbares, welches in hellgelben hexagonalen Tafeln kristallisiert und dem Oele den eigenartigen Geruch mitteilt. Der Glyzeringehalt beträgt $11,4 \%$. Verseifungszahl 209,3, Hehnerzahl 91,75, Jodzahl 81,44, Reichert-Meissl'sche Zahl 1,54. An Säuren enthält das Oel Palmitinsäure 22,0\%, Oleïn- and Linolensäure 73,6\%, Caprin-, Capron- und Caprylsäure 3,0\%, Schmelzpunkt der Fettsäuren $38^{\circ}$. (J.A m. Chem. Soc. 1902, 771.) -A-

Einwirkang des Bleichens anf Bienenwachs. Von Ragnar Berg. Da es oft vorgekommen ist, dass ein Wachs vor dem Bleichen als rein, aber nach dem Bleichen, da die Sänrezahl ungewöhnlich hoch und demnach die Verhältniszahl ungewohnlich niedrig war, als stearinhaltig befunden wurde, so hat Verf. verschiedene Wachse ungebleicht und gebleicht untersucht and die Resultate in 2 Tabellen niedergelegt. Daraus ergiebt sich folgendes: Die Säurezahl wird stets vergrössert, und zwar wie zu erwarten ist, am wenigsten bei der Naturbleiche und am meisten bei der Chromsänrebleiche. Bei der Naturbleiche, gewöhnlich auch bei der Permanganatbleiche, bleibt die Esterzahl unverändert oder wird gar erhøht, während bei der Chromsäurebleiche stets Verminderung derselben darch Hydrolyse stattfindet. Die Vergrösserung der Säurezahl ist stets so gross, dass das Verhältn is $z$ wischen Säurezahl nud Esterzahl herabgedrückt wird, oft sogar um ein so Beträchtliches vermindert wird, dass man, wenn keine qualitative Probe vorgenommen wird, das Wachs als mit Stearin versetzt anzusprechen sich wohl berechtigt fühlen konnte. Eine weitere Folge ist, dass die Verseifungszahl bei dem gebleichten Wachse stets eine Kleinigkeit höher als bei dem gelben gefunden wird. Bei fast allen mit Chromsäare gebleichten Wachsen wurde die Jodzahl erhöht. Nur die auch sonst ziemlich merkwürdigen italienischen Wachssorten erleiden eine Erniedrigung ihrer Jodanfnahmefähigkeit, ebenso alle Natur- oder durch Permanganat gebleichten Wachse. Die Buchnersche Zahl wird durch die Natur- sowie durch die Permanganatbleiche ein wenig herabgesetzt. Bei der Chromsäurebleiche wird diese Herabsetzung durch die tiefgehende Hydrolyse nicht nur aufgehoben, sondern in den meisten Fällen sogar in einen Ueberschuss verwandelt. Aach hierbei bilden aber die italienischen Wachssorten eine Ausnahme, indem trotz der hohen Säurezahl die Buchnersche Zahl, sich gleich bleibt oder gar sinkt. 